\title{
Michel Marc Bouchard : adapté ou transcréé ?
}

\author{
Entretien accordé à Marie Pascal, Université Dalhousie
}

Dramaturge québécois de renom, Michel Marc Bouchard est connu pour sa réflexion sur des personnages marginaux et sur les difficultés de la communication. D'après le site officiel de l'auteur (https://www.michelmarcbouchard.com/), ses œuvres les plus connues sont : Les feluettes (Lilies), Les muses orphelines (The Orphan Muses), Sous le regard des mouches, Le peintre des Madonnes (The Madonna Painter), Tom à la ferme (Tom at the Farm), Christine, la reine-garçon (Christina, The Girl King) et, dernièrement, La nuit où Laurier Gaudreault s'est réveillé. La plupart des titres susmentionnés ont été traduits en plusieurs langues et certains ont vu le jour au grand écran, ce pourquoi il m'a semblé particulièrement intéressant de le questionner à nouveau (plusieurs autres entretiens portent sur son avis concernant les adaptations les plus anciennes) sur ce phénomène visant à porter un texte de théâtre au cinéma.

\section{Son ouvre}

M.P. On dirait que vos pièces tournent souvent autour de la question de la marginalité. Pourquoi des personnages rejetés et martyrisés ?

M.M.B. Si on revient un peu dans le temps (je suis quand même né à la fin des années 1950), avant même qu'il n'existe de vocabulaire pour les décrire, les homosexuels étaient extrêmement marginalisés, dans le sens qu'ils devaient rester secrets, qu'ils se ghettoïsaient facilement pour pouvoir assouvir leur amour et leurs désirs. Ils avaient une vision assez marginale des choses, autrement dit, un point de vue sur le monde, la réalité, les autres, qui leur était particulier. Je ne revendique pas le fait que la marginalité appartienne uniquement aux homosexuels et j'ai au contraire montré dans mon travail que mes personnages n'étaient pas nécessairement définis par leur orientation sexuelle. Il n'en demeure pas moins que ce qui m'a appelé à voir le monde différemment, c'est que j'étais différent de ce monde-là. J'ai été élevé dans un univers catholique très conservateur, rural en plus. Je n'ai pas eu d'autre choix que de fabuler, de me réinventer un autre univers afin d'exister. C'était une forme d'évasion, une fantaisie. J'ai dit dans Tom à la ferme que les homosexuels devaient apprendre à mentir avant d'apprendre à aimer. De cette façon, ils apprenaient à écrire, à fabuler, à fantasmer leur propre désir. Ce qui fait que, dans mon œuvre, les marginaux sont très présents mais il en est de même de leur affirmation, elle-aussi très importante dans la démarche.

\section{M.P. Le théâtre peut-il être vu comme un mode d'expression de cette marginalité ?}

M.M.B. C'est d'une construction qu'il s'agit et c'est bien de le faire au théâtre parce que le théâtre est marginal par rapport à la société. Ce qui fait qu'il est plus apte, prêt et gourmand de l'expression d'une marginalité, d'un affranchissement de la marginalité. Le théâtre est un bon lieu pour représenter la marginalité, non seulement parce qu'il est marginal par rapport aux autres formes mais aussi parce que c'est un lieu de l'argumentaire.

\section{M.P. L'histoire d'amour qui finit bien est-elle un mythe pour vous ?}

M.M.B. Je pense que l'histoire d'amour qui finit bien, dans la création littéraire et théâtrale, a quelque chose de profondément rassurant. J'ai utilisé, à un moment donné, un terme plus radical : je disais «commercial», car je pensais que ça manquait, quelque part, d'une position éditoriale. On aura tout à l'heure à parler de Tom à la ferme parce que les fins sont diamétralement opposées entre ma pièce et le film de Xavier Dolan, mais j'aimerais dire que dans le processus d'écriture de Tom à la ferme, j'ai fait quelque chose que je ne referai plus 
jamais car je l'ai trouvé un peu déstabilisant pour l'équipe : quand on a commencé à répéter, je n'ai pas apporté la fin... La création d'une pièce, c'est un étranger, et lorsque tu la mets en 3D, dans l'espace, avec les acteurs, c'est un étranger que tu vas devoir apprivoiser. Tom à la ferme, je n'arrivais pas à la terminer. Et au bout d'un mois, je suis arrivé avec cette fin-là. La production a été étonnée mais moi, j'avais l'impression qu'il fallait qu'on finisse comme ça, sur la question : «Pourquoi Tom en arrive à ça ?» [Tom tue Francis, le frère de son amant défunt, dont il est progressivement tombé amoureux.] Au final, Tom se tue beaucoup plus qu'il tue Francis : il tue en lui un désir toxique, malsain, violent, masochiste. Dans les trois fins que j'avais écrites, il y avait une fin heureuse, celle où ils décidaient de rester ensemble. Avec cette fin, je me suis dit : «Tu laisses rien au monde, tu prends tout ». J'aime bien que les fins, que les histoires d'amour, ne se terminent pas nécessairement bien. Mais dans ce caslà, c'est radical. Dans Les feluettes, c'est une approche beaucoup plus opératique, une approche qui se veut boucler toute l'histoire de d'Annunzio, boucler l'histoire de Saint Sébastien, boucler toute leur vie : il n'a pas d'échappatoire en 1912, il n'en a pas. [Simon et Vallier décident de se suicider ensemble, à l'instar d'une pièce de d'Annunzio qu'ils ont jouée pour le spectacle de leur école.] Je voulais magnifier leur suicide. Sauf que ce qui sera leur véritable fin, c'est que quelqu'un intervient et n'en sauve qu'un [Bilodeau sauve son idole, Simon, et laisse Vallier périr dans les flammes] et le condamne à une vie plus misérable que la mort à laquelle il aspirait. Ce choc-là, de ces fins qui sont noires et brutales, permet une véritable discussion après. Ça laisse une empreinte plus forte que l'histoire d'amour qui finit bien. Et n'oublions pas que nous sommes au théâtre et que, dans trois minutes, ils viendront saluer devant vous. Ils ne sont pas morts : ce sont des morts métaphoriques.

\section{M.P. Pour qui écrivez-vous ?}

M.M.B. Pour moi. C'est sûr qu'avec le temps, j'ai pris conscience du rapport organique entre la salle et la scène, et que je suis devenu très attentif au public. Je suis capable de sentir le public même si j'écris ici [chez lui], mais je le fais avant tout pour moi : si cela ne me fait pas rire, si cela ne me touche pas, si cela ne me provoque pas, pourquoi est-ce que je donnerais ça au public? Je trouve que l'écriture, c'est un voyage et j'aime bien ne pas savoir nécessairement où je m'en vais quand je commence. Je ne fais pas de sketch, je ne fais pas de construction. Les premières pages sont comme un chantier et là, je suis capable de raconter l'histoire. Maintenant que j'ai le luxe du temps, je peux me permettre de me tromper, je peux aller vers quelque chose, je peux changer un peu le destin. C'est d'ailleurs ce qui est arrivé avec Tom à la ferme: Francis était un personnage qui parlait peu, qui ne parlait pas. Je travaillais un personnage presque muet. Mais par couches, il s'est mis à parler.

Mais j'écris également pour les comédiens. On a fait La nuit où Laurier Gaudreault s'est réveillé, cette année, au TNM [le Théâtre du Nouveau Monde] et ça faisait deux ans et demi que l'équipe d'acteurs était choisie. On s'est rencontré cinq ou six fois pendant le processus, juste pour entendre le texte. Personnellement, je n'aime pas recevoir des idées mais j'aime recevoir des questions qui proposent une dynamique. C'est pour ça que, quand on commence les répétitions, je suis là la première semaine pour entendre les questions et je peux, lors des lectures, montrer mon opposition. Pendant la première semaine seulement parce qu'on est en train d'établir des bases fondamentales. Après, je disparais, je laisse faire le metteur en scène car c'est lui le chef dans la salle de répétition. Je ne reviens que les dix derniers jours. Ce n'est que récemment que je commence à écrire en pensant aux acteurs. En dirigeant mes propres créations, je me suis rendu compte que ce qui n'est pas bien, pour les acteurs, c'est quand le metteur en scène (qui est aussi l'auteur) sait tout. Dans le métier d'acteur, je pense qu'il y a une démarche de qualité de recherche qui est fondamentale et, si l'auteur impose à chaque fois ses réponses, je trouve que c'est pas toujours d'à propos. 


\section{Sur Les feluettes :}

M.P. Je sais que vous vous êtes beaucoup exprimé sur Les feluettes mais il me reste encore quelques questions auxquelles je ne crois pas que vous ayez répondu. Tout d'abord, est-il nécessaire que l'amoureux de Simon soit (1) français et (2) comte ?

M.M.B. Français parce que, pour les Québécois, le romantisme et le langage sont tous deux d'importance et c'est pas quelque chose qu'on a ici. Donc, pour moi, c'était fondamental qu'on ait un personnage français. Ce qui m'a aidé aussi, c'est que cette histoire est presque plausible parce qu'à cette époque-là, à Roberval, il y avait une communauté d'aristocrates français. Si bien que moi qui suis tombé sur cette monographie de la ville de Roberval pendant la période rose, j'ai eu envie de ramener ici, pour cette histoire d'amour entre deux hommes, des éléments de romans de gare, de téléromans un peu fleur bleue, dans un décor qui faisait que cette histoire-là était plausible. D'où l'hôtel, les Français... Il y a aussi le rapport à quelque chose qui est tellement loin de nous, l'aristocratie, une aristocratie complètement déjantée (Vallier et sa mère n'ont plus rien d'autre que le titre). J'y tenais depuis le début qu'il soit Français parce que ça devait être un clash au niveau du langage, au niveau de l'expression des sentiments, au niveau de la littérature, au niveau des référents. Simon parle avec émotion, Vallier raisonne son émotion. Et comte donc, pour lui donner un côté à la fois désuet et presque mythique. Vallier, c'est un comte, c'est un prince.

\section{M.P. Accepteriez-vous de vous exprimer sur le personnage de Bilodeau ?}

M.M.B. Bilodeau qui, dans la première mouture du Feluette, n'était qu'un petit jaloux, a ensuite pris beaucoup plus d'ampleur étant donnée son importance dans la structure et dans le deuxième niveau de lecture. À travers Bilodeau, je faisais à l'époque un commentaire sur le fait que les homosexuels ne sont pas qu'une «race». Il y a plusieurs «races» d'homosexuels, et là, il y a celle de Bilodeau, notre pire ennemi alors qu'il devrait être notre meilleur ami. Tragiquement, par ce rejet de l'amour de la part de Simon, il devient vilain, un peu comme le troisième membre du couple qui n'accepte pas le refus et qui détruit les autres en empêchant le geste de passion, le pacte final. C'est un personnage que les gens aiment beaucoup dans sa méchanceté et dans sa manière de dire les choses. Mais c'est aussi un personnage pitoyable, à la merci de sa mère et de Mme Lavigne. C'est un personnage qui a été très agréable à écrire mais qu'il fallait aussi doser. Ce n'est pas pour rien qu'il est devenu curé... Les Anglais ont tendance à monter la pièce de façon anticléricale mais ce n'est pas là que je suis allé : Bilodeau se sert d'un costume pour cacher ses passions, ce n'est pas une charge contre l'Église. En effet, le Père Saint-Michel est au contraire un très bon prêtre. Bilodeau est arrivé à cet échelon de l'épiscopat parce que c'est un fou de religion; ses menaces sont celles d'un jaloux qui se sert de la caution sociale pour posséder les amants ou, du moins, pour posséder Simon.

\section{M.P. Que pensez-vous de l'adaptation cinématographique de John Greyson ?}

M.M.B. C'est un film un peu iconoclaste que j'aime beaucoup. On a eu de la chance d'avoir au TIFF [Toronto International Film Festival], à Toronto, un événement autour de John Greyson après son retour d'incarcération en Égypte. Il y a eu une espèce de soirée gala pour remastériser Les feluettes. C'était touchant de voir ça après genre vingt ans.

\section{M.P. Que dire de la traduction du titre (de Feluettes, il devient Lilies) ?}

M.M.B. C'est drôle que tu me poses la question parce que hier, on parlait de l'anthologie de mon œuvre qui va paraître en avril et on discutait justement du titre des Feluettes, qui n'est pas un titre simple. Pour «Lilies », le chemin qui a été fait, c'est qu'aucun des termes comme « sissy », ces noms vulgaires, ne fonctionnait. Il n’y avait pas vraiment d'archaïsme pour ça. 
Linda Gaboriau, qui a traduit presque toutes mes pièces en anglais, est allée puiser dans l'univers d'Oscar Wilde, d'où «Lily White ». Elle trouvait que l'époque était intéressante, puisqu'on est presque à la fin du dix-neuvième, et elle voulait faire un rappel. Ce qu'il y a d'intéressant dans «Lilies », c'est la France, c'est le Québec, et aussi, dans plusieurs pays, c'était ce qu'on tatouait sur le bras des prisonniers. J'ai oublié de dire en plus de ça que c'est la fleur des catholiques.

\section{M.P. À votre avis, le fait d'avoir des hommes qui jouent des femmes dans les passages analeptiques marche-t-il aussi bien au cinéma qu'au théâtre ?}

M.M.B. Oui, parce que le film l'avoue. Il y a une représentation en prison, ce qui fait qu'on accepte cette convention-là. Dès qu'on arrive à la scène du confessionnal, c'est comme si on était transporté dans l'imaginaire du récit. Cela en fait un film assez iconoclaste du fait que tous les rôles sont joués par des hommes sans qu'on en abuse comme dans un drag-queen show. On est vraiment dans des personnages qui ont des affects, un vrai parcours narratif, et qui ne sont pas juste là pour porter une robe... ce qui est parfois un problème avec les représentations de la pièce. Nous, à la création, il n'y avait pas de robe : c'était une façon de se comporter, une façon de porter une chemise, une façon de parler, etc. La robe, c'est un accessoire. Cette idée d'hommes qui jouent des femmes m'est venue lors de mes études à l'université d'Ottawa, au début des années 1980. On était, surtout dans la création, dans un discours féministe radical où on disait que les hommes avaient trahi les femmes au théâtre depuis qu'ils écrivent le théâtre... Moi je me suis dit que j'allais faire des femmes écrites par un homme pour des hommes. Les feluettes, ça a été écrit pour des hommes.

\section{Sur les adaptations}

\section{M.P. Avez-vous regretté des altérations ?}

M.M.B. Dans Les feluettes, je regrette la fin du film et le fait que ce soit Bilodeau qui commette le crime alors que dans la pièce, c'est le pacte de suicide que Bilodeau empêche. Avec Tom à la ferme, la réponse est un peu la même. On peut négocier beaucoup de choses dans le parcours mais la position éditoriale, c'est très difficile, la fin, c'est vraiment quelque chose... J'ai cheminé avec eux [John Greyson et Xavier Dolan] comprenant que c'étaient les fins qu'ils voulaient. Je suis plus en harmonie avec celle de Lilies par contre. Dans Tom à la ferme, il n'y a pas de dimension de genre. Je pense que Xavier l'a encore plus marqué dans le film. C'est très angoissant, on ne sait pas quand Francis va surgir, ce qui va arriver à Tom et, soudainement, il y a une espèce d'apaisement qu'on ne comprend pas. Parce que, à la fin, quand ils sont vers cet étang, tout est là pour que la scène de l'assassinat de Francis se passe... et il a peur... C'est même mieux représenté dans ce que Xavier [Dolan] amorce que ce qu'il se passe au théâtre mais ça ne se passe pas... Mais Xavier [Dolan] disait « Je ne suis pas là pour dire aux gays 'ça marche pas, tuez l'autre'... Je suis pas capable!» John [Greyson] avait dit: «Je ne suis pas là pour dire aux adolescents 'tuez-vous'... » Donc tu vois, il y a deux positions là-dedans qui sont deux positions très politiques.

En plus, ce que Xavier a fait, c'est que toute la scène de l'aveu disparait... En fait, Tom à la ferme est de l'ordre de la tragédie parce que Tom arrive dans une histoire qui a déjà commencé bien avant lui et dont il sera la prochaine victime, donc il y a vraiment un fatum. Ce que j'aime, dans ma pièce, c'est le fait que ce soit Francis qui avoue, qui se confie sur la pire chose qu'il a faite [Francis avoue à Tom avoir défiguré le premier amoureux de son frère décédé]. Après avoir dit tout ça, il demande à Tom de rester. Dans la nouvelle construction, celle de Dolan, on a cet aveu à la fin par le barman. Je me demande pourquoi il ne l'a pas laissé dire par Francis. 
Là, j'ai appris que ça allait paraître dans les journaux demain donc je peux te le dire : Xavier [Dolan] va adapter mon dernier texte [La nuit où Laurier Gaudreault s'est réveillé]. On s'en est parlé samedi et là, je lui ai demandé de me dire ce qu'il ne prenait pas. Les deux ou trois choses qu'il ne prend pas, je comprends et ce n'est pas dommageable. Il n'en demeure pas moins que lorsque l'auteur est vivant, qu'on est ami, c'est plus difficile car on peut dire : «c'est ça ou rien! »

\section{M.P. Quelles raisons expliquent-elles que vos textes soient si souvent adaptés ?}

M.M.B. J'y répondrai de la même façon que « pourquoi sont-ils si rejoués » ? Il y a l'histoire, il $\mathrm{y}$ a les personnages. On est dans un monde où on veut des récits. Si le récit au théâtre peut permettre l'évocation au public, j'imagine que c'est la même chose pour les gens qui veulent m'adapter, c'est qu'ils ont vécu ce moment de révélation-là. Je suis un conteur. Il y a certes au cinéma un récit qui doit être raconté d'une autre façon et c'est clair qu'au niveau du récit, le cinéma sera perdant par rapport au théâtre. Car le temps au théâtre n'a pas du tout la même valeur qu'au cinéma. Dans un récit, je peux raconter trois millions de choses mais le récit au cinéma n'a pas (à moins que le narrateur soit avoué depuis le début, avec la voix-off) la possibilité de dire tout ce que le récit dira. La première chose que j'ai demandée à Xavier c'est «qu'est-ce que tu abandonnes ? » car oui, il faut qu'il ait abandonné quelque chose. Même chose pour toute adaptation ratée de roman, c'est qu'on a voulu trop en garder. Moi, j'avais proposé à Xavier qu'il révèle tout l'inconscient de Tom, parce qu'on l'a, dans la pièce. On aurait pu avoir, autrement dit, Tom en voix-off pendant qu'il joue. On a cette possibilité au cinéma, celle de la voix-off. Des fois ça marche et des fois c'est très mauvais. Mais ici, ce qui est beau avec le récit de Tom, c'est que c'est au public d'aller démêler ce qu'il dit vraiment de son inconscient. C'est une approche stylistique qui revient à dire : «Vous, public, faites le ménage ». Par ailleurs, en plein milieu de la pièce, il n'y a plus de non-dit, il n'y a plus d'introspection. Et après, l'introspection revient, c'est le grand thème de la fin. Et comment tu fais ça au cinéma ? Dès le départ, il a dit qu'il ne le ferait pas.

\section{M.P. Quelles sont les difficultés d'être adapté ? De devoir lâcher prise vis-à-vis de votre œuvre?}

M.M.B. Le sentiment que t'as... Il y a quelqu'un dans ma maison. Il y a quelqu'un qui change la couleur des murs, la place des fauteuils... et ça prend ma maison! Il n'en demeure pas moins que, pour moi, le cinéma n'est pas le point ultime de ma création. Ça reste la pièce. La pièce vit, revit, on peut la relire, la revoir, ça se passera demain. C'est ça qui est rassurant. C'est pas comme si j'avais écrit un roman qui n'avait pas beaucoup marché. Là, ce serait un peu difficile. Et dans les deux cas qui nous intéressent aujourd'hui, on a travaillé ensemble avec les réalisateurs. Il y a eu des aventures beaucoup plus difficiles... L'adaptation de Christine, la reine-garçon a par exemple été un cauchemar, avec un résultat qui n'avait absolument rien à voir avec ce que j'avais pu écrire. J'ai beaucoup travaillé, j'ai fait beaucoup de recherches pour offrir un scénario ; le réalisateur a eu, pour moi, la mauvaise idée de refuser pour aller vers un scénario plus proche du biopic. Moi, pendant le processus d'écriture du scénario, j'ai écrit la pièce. J'ai mis mon point de vue, un point de vue qui n'était pas partagé par le réalisateur. La pièce a été publiée et jouée avant la sortie du film. Quand le film est sorti, la pièce avait déjà sa renommée (pour ici). Ce qui fait que j'avais au moins pu, en tant que créateur, poser le geste de l'éditorial. Peut-être aussi le fait que je sois un homme de théâtre, pas un homme de roman, ça m'aide à laisser un peu ma pièce parce que le théâtre est un art de l'abandon. C'est pas comme une recette de gâteau où, sur la boîte, il y a toutes les étapes bien définies. Dans une pièce de théâtre, il y a des trous. 


\section{M.P. Quels sont finalement les aspects qui rendent mieux au cinéma ?}

M.M.B. Je suis un homme de théâtre. Je pense qu'il y a, au cinéma, des choses qui peuvent nous toucher, des choses mémorables, mais il n'y a rien comme une production mémorable au théâtre. Il y a des gens qui me parlent encore d'expériences qu'ils ont vécues au théâtre... ça se passe là. C'est viscéral, ça se passe là. Ils ont eu du vrai, en continu, pas en montage. À travers cette relation, on est parfois troublé dans une interprétation, on a l'impression d'être touché par un acteur. Un ensemble. 